\title{
Identification of COMMD1 as a novel lamin A binding partner
}

\author{
ZHIWEN JIANG ${ }^{1,2}$, WEICHUN CHEN ${ }^{1,3}$, JING ZHOU ${ }^{1,2}$, QI PENG ${ }^{1,2}$, HUILING ZHENG $^{1,2}$, YUAN YUAN $^{1,2}$, \\ HONGJING CUI ${ }^{1,2}$, WEI ZHAO ${ }^{1,2}$, XUERONG SUN $^{1,2}$, ZHONGJUN ZHOU $^{4}$ and XINGUANG LIU ${ }^{1-3}$ \\ ${ }^{1}$ Institute of Aging Research, Guangdong Provincial Key Laboratory of Medical Molecular Diagnostics; \\ ${ }^{2}$ Dongguan Scientific Research Center; ${ }^{3}$ Institute of Biochemistry and Molecular Biology, Guangdong Medical University, \\ Dongguan, Guangdong 523808; ${ }^{4}$ Department of Biochemistry, Li Ka Shing Faculty of Medicine, \\ The University of Hong Kong, Hong Kong, SAR, P.R. China
}

Received August 12,2018; Accepted May 20, 2019

DOI: $10.3892 / \mathrm{mmr} .2019 .10419$

\begin{abstract}
Lamin A, which is encoded by the LMNA gene, regulates gene expression and genome stability through interactions with a variety of proteins. Mutations in LMNA lead to a diverse set of inherited human diseases, collectively referred to as laminopathies. To gain insight into the protein interactions of lamin A, a yeast two-hybrid screen was conducted using the carboxy-terminus of lamin A. The screen identified copper metabolism MURR1 domain-containing 1 (COMMD1) as a novel lamin A binding partner. Colocalization experiments using fluorescent confocal microscopy revealed that COMMD1 colocalized with lamin A in 293 cells. Furthermore, the COMMD1-lamin A protein interaction was also demonstrated in co-immunoprecipitation experiments. Collectively, the present study demonstrated a physical interaction between COMMD1 and lamin A, which may aid to elucidate the mechanisms of lamin $\mathrm{A}$ in the aging process.
\end{abstract}

\section{Introduction}

Lamin A, which is encoded by the LMNA gene, is a major component of the nuclear lamina and nuclear skeleton (1). Lamin A is expressed in most adult tissues $(2,3)$, with the expression of lamin $\mathrm{A}$ increasing with age in somatic cells (4). Although studies have revealed that lamin A serves a structural role during interphase (5), lamin A is increasingly recognized as a mediator, and possibly a regulator, of nuclear

Correspondence to: Dr Xinguang Liu, Institute of Aging Research, Guangdong Provincial Key Laboratory of Medical Molecular Diagnostics, Guangdong Medical University, 1 Xincheng Boulevard, Dongguan, Guangdong 523808, P.R. China

E-mail: xgliu@gdmu.edu.cn

Abbreviations: HGPS, Hutchinson-Gilford progeria syndrome; COMMD1, copper metabolism MURR1 domain-containing 1; GFP, green fluorescent protein; RT-PCR, reverse transcription polymerase chain reaction; $N F-\kappa B$, nuclear factor- $\kappa \mathrm{B}$; $\mathrm{ARF}$, alternate reading frame protein product of the CDKN2A locus

Key words: lamin A, aging, interaction, COMMD1 processes through its interactions with a variety of nuclear factors, including double-stranded DNA, transcriptional regulators, nuclear membrane-associated proteins and nuclear pore complexes $(6,7)$. Dysfunction of lamin A interrupts chromatin organization, the DNA damage response, telomere maintenance, cellular senescence and apoptosis $(8,9)$. Mutations in the LMNA gene cause a heterogeneous group of human diseases that are collectively termed laminopathies, including progeroid syndromes and premature aging disorders that primarily affect striated muscle, adipose, bone and neuronal tissues, such as Hutchinson-Gilford progeria syndrome (HGPS) $(6,10,11)$. Mutations leading to laminopathies are distributed throughout the LMNA gene and show a high degree of tissue specificity $(3,12)$. How mutations in $L M N A$ cause disease and why laminopathies are highly tissue-specific remain unclear (3). Nuclear envelope proteomes are highly variable among tissues $(13,14)$. Additionally, variants of lamin A may interact differently with proteins that are themselves expressed in a tissue-specific manner, which could explain the tissue specificity of laminopathies (15). However, determining the molecular mechanisms underlying changes in lamin A protein interactions remains a clinical challenge.

The G608G mutation in the $L M N A$ gene causes a truncation of lamin A, with a 50-residue region lost that includes a second proteolytic site for zinc metallopeptidase STE24 (ZMPSTE24), resulting in an unprocessed prelamin A termed progerin in patients with HGPS (16). The accumulation of truncated lamin A in HGPS impedes the release of proteins from the nuclear membrane and disrupts their regulatory functions, thereby accelerating a subset of pathological changes that contribute to the aging processes $(17,18)$. Notably, mice carrying lamin A mutations also exhibit symptoms consistent with HGPS, including the thinning of skin, hypoplasia, the degeneration of cardiac and skeletal muscles, and osteoporosis (19). Increased levels of wild-type lamin A in normal human cells result in a decreased replicative lifespan and nuclear membrane alterations that lead to phenotypic changes similar to those observed in HGPS fibroblasts $(13,14)$. These studies suggest that wild-type lamin A, similar to mutated $\operatorname{lamin} \mathrm{A}$, is also involved in the aging processes.

To improve understanding of the pathological mechanisms involved in laminopathies and the aging process, the present 
study sought to systematically identify lamin A-interacting proteins in an unbiased manner. A yeast two-hybrid screen of a human skeletal muscle cDNA library was performed using the carboxy (C)-terminus of lamin A as bait to search for novel lamin A-interacting factors. This screening identified copper metabolism MURR1 domain-containing 1 (COMMD1, formerly known as MURR1) as a novel binding partner of lamin A. Their binding affinity was further validated using confocal colocalization and co-immunoprecipitation experiments.

\section{Materials and methods}

Yeast two-hybrid analysis. Yeast two-hybrid analysis was conducted using a GAL4-based system to screen a human skeletal muscle complementary DNA (cDNA) library (Matchmaker GAL4 two-hybrid system; Clontech Laboratories, Inc.). Briefly, a bait protein was constructed by cloning the C-terminus of lamin A (mRNA sequence 1,413-2,241) in frame with the GAL4 binding domain using the EcoRI and BamHI restriction sites of the pGBKT7 vector (Clontech Laboratories, Inc.). The yeast Saccharomyces cerevisiae strain AH109 (Clontech Laboratories, Inc.) was sequentially transformed with the C-terminal lamin $\mathrm{A}$ bait vector (pGBKT7-LA-C; Clontech Laboratories, Inc.) and the Matchmaker human skeletal muscle cDNA library (Clontech Laboratories, Inc.) cloned into pACT2 (Clontech Laboratories, Inc.) according to the manufacturer's protocol (Clontech Laboratories, Inc.). Saccharomyces cerevisiae strain AH109 transformed with pCL1 (encodes the full-length and wild-type GAL4 protein) vector was provided as positive control. Transformants were plated on synthetic defined (SD)/histidine/leucine/tryptophan (TDO) medium (Clontech Laboratories, Inc.) (low-stringency protocol); a total of $2 \times 10^{7}$ colonies were screened. Colonies were transferred to SD/adenine/histidine/leucine/tryptophan (QDO) plates (Clontech Laboratories, Inc.) containing 5-bromo-4-chloro3 -indolyl- $\alpha$-D-galactopyranoside (X- $\alpha$-Gal) following two rounds of selection. Positive clones were identified under high-stringency conditions and were defined as clones that exhibited growth on the QDO plates that were strongly positive for galactosidase activity. The selected clones were further analyzed by Sanger sequencing (Sangon Biotech Co. Ltd.) and compared with known sequences in GenBank using a BLAST search (https://blast.ncbi.nlm.nih.gov/Blast.cgi).

Cell culture. The 293 cell line (Stem Cell Bank; Chinese Academy of Sciences) was cultivated in DMEM (Gibco; Thermo Fisher Scientific, Inc.) supplemented with $10 \%$ fetal calf serum (Gibco; Thermo Fisher Scientific, Inc.), $100 \mathrm{U} / \mathrm{ml}$ penicillin and $100 \mu \mathrm{g} / \mathrm{ml}$ streptomycin. Serial passaging was performed when the cells reached a confluence of $80 \%$.

Western blotting. Whole cell extracts were prepared using RIPA buffer [50 mM Tris- $\mathrm{HCl}$ (pH 7.4), $150 \mathrm{mM}$ sodium chloride, $1 \%$ NP-40, $0.5 \%$ sodium deoxycholate, $0.1 \%$ SDS, $1 \mathrm{mM}$ phenylmethylsulfonyl fluoride, $1 \mu \mathrm{g} / \mathrm{ml}$ leupeptin and $1 \mu \mathrm{g} / \mathrm{ml}$ pepstatin]. Protein was quantified using a bicinchoninic protein assay kit (Pierce; Thermo Fisher Scientific, Inc.). Subsequently, 10-20 $\mu \mathrm{g}$ protein was loaded into each lane. Proteins were separated by $12 \%$ SDS-PAGE. Subsequently, gels were blotted onto PVDF membranes. The PVDF membranes were blocked for $1 \mathrm{~h}$ at room temperature $\left(25^{\circ} \mathrm{C}\right)$ in Tris-buffered saline containing $0.1 \%$ Tween 20 and $5 \%$ non-fat milk. Primary antibodies were diluted in blocking solution and incubated with the membranes overnight at $4^{\circ} \mathrm{C}$. Anti-COMMD1 antibody (1:1,000; cat. no. sc-166248; Santa Cruz Biotechnology, Inc.), anti-lamin A/C antibody (1:2,000; cat. no. sc-20681; Santa Cruz Biotechnology, Inc.), anti-hemagglutinin (HA) antibody (1:1,000; cat. no. 11867423001; Roche Diagnostics) and anti-Flag antibody (1:1,000; cat. no. F3165; Sigma-Aldrich; Merck KGaA) were used as primary antibodies. Anti-GAPDH antibody (1:1,000; cat. no. 60004-1-Ig; ProteinTech Group, Inc.) was used to detect the protein expression level of the loading control GAPDH. Secondary antibodies horseradish peroxidase (HRP)-conjugated goat anti-rabbit (cat. no. A120-101P; Bethyl Laboratories, Inc.), goat anti-rat (cat. no. A110-143P; Bethyl Laboratories, Inc.) and goat anti-mouse (cat. no. AP308P; Merck KGaA) were diluted at 1:5,000 and incubations were performed for $1 \mathrm{~h}$ at room temperature $\left(25^{\circ} \mathrm{C}\right)$. The proteins were detected using a western chemiluminescent HRP substrate (EMD Millipore).

Fluorescence confocal microscopy. Total RNA was extracted from 293 cells using TRIzol reagent (Invitrogen; Thermo Fisher Scientific, Inc.). The first strand cDNA was synthesized using the PrimeScript $1^{\text {st }}$ Strand cDNA Synthesis kit (Takara Bio, Inc.) following the manufacturer's protocol. COMMD1 coding sequence (CDS) and Lamin A CDS were synthesized using the same first strand cDNA templates. COMMD1 CDS was synthesized using the primers forward, 5'-CCCTCGAGA TGGCGGGCGAGCTTGAG-3' and reverse, 5'-CGGAAT TCGGTTAGGCTGGCTGATC-3'. PCRs were performed using the rTaq DNA Polymerase (Takara Bio, Inc.) in a total volume of $20 \mu \mathrm{l}$ and amplification protocol consisted of an initial denaturation at $94^{\circ} \mathrm{C}$ for $5 \mathrm{~min}$, followed by 30 cycles of denaturation for $45 \mathrm{sec}$ at $94^{\circ} \mathrm{C}$, annealing for $45 \mathrm{sec}$ at $60^{\circ} \mathrm{C}$ and extension for $1 \mathrm{~min}$ at $72^{\circ} \mathrm{C}$, followed by a final extension at $72^{\circ} \mathrm{C}$ for $10 \mathrm{~min}$. The green fluorescent protein (GFP)-COMMD1 vector was generated by inserting COMMD1 CDS into the pEGFP-N1 vector (Clontech Laboratories, Inc.), which contains a GFP expression cassette, with XhoI and EcoRI restriction sites. Lamin A CDS was generated with the following thermocycling conditions: Initial denaturation at $94^{\circ} \mathrm{C}$ for $5 \mathrm{~min}$, followed by 30 cycles of denaturation for $45 \mathrm{sec}$ at $94^{\circ} \mathrm{C}$, annealing for $1 \mathrm{~min} \mathrm{sec}$ at $60^{\circ} \mathrm{C}$ and extension for $90 \mathrm{sec}$ at $72^{\circ} \mathrm{C}$, followed by a final extension at $72^{\circ} \mathrm{C}$ for $10 \mathrm{~min}$. Lamin A CDS was amplified using the primers forward 5'-ACGCTCGAGATGGAGACC CCGTCCCAGCGGC-3' and reverse 5'-CGGGATCCGCGG GCTCTGGGTTCGGGGGCT-3'. The red fluorescent protein (RFP)-lamin A vector was generated by inserting lamin A CDS into the pDsRed2-N1 vector (Clontech Laboratories, Inc.), which contains an RFP expression cassette, using the $\mathrm{XhoI}$ and $\mathrm{BamHI}$ restriction sites. All sequences from all plasmids were confirmed by DNA sequencing following cloning.

The day prior to transfection, 293 cells $\left(2 \times 10^{5}\right)$ were seeded into confocal dishes. When the cells reached $\sim 80 \%$ confluence, $2.5 \mu \mathrm{g}$ pEGFP-N1-COMMD1 and $2.5 \mu \mathrm{g}$ pDsRed2-N1 or $2.5 \mu \mathrm{g}$ pDsRed2-N1-lamin A were transfected into cells using Lipofectamine ${ }^{\circledR} 2000$ transfection reagent (Invitrogen; 
A

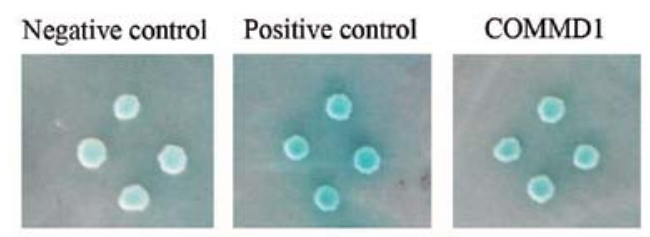

C

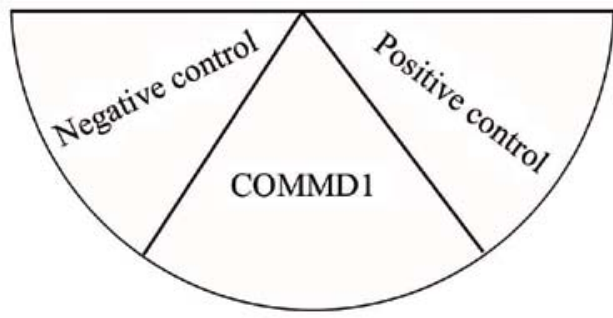

B

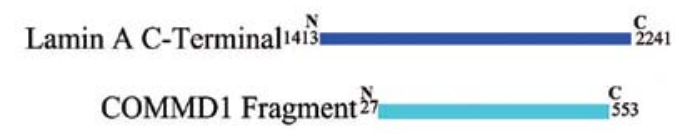

D

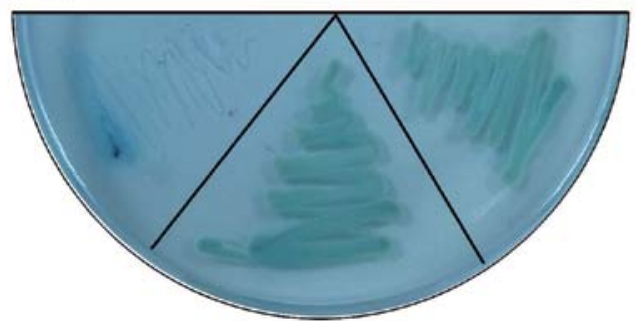

Figure 1. Analysis of the COMMD1-lamin A interaction using a yeast two-hybrid assay. (A) Positive clones interacting with the C-terminus of lamin A on low-stringency synthetic defined/histidine/leucine/tryptophan plates containing X- $\alpha$-gal (blue). (B) Schematic diagram of the interaction between the COMMD1 sequence and the C-terminus of lamin A. (C) Schematic diagram of the second round selection using a high stringency protocol. (D) Diploid AH109 yeast were re-streaked onto synthetic defined/adenine/histidine/leucine/tryptophan plates containing X- $\alpha$-gal to test for an interaction. AH109 yeast transformed with the C-terminus of lamin A in the pGBKT7 vector. Empty pGBKT7-LA-C was used as a negative control. AH109 yeast transformed with pCL1 was used as positive control. A blue signal indicates the activation of the reporter genes. COMMD1, copper metabolism MURR1 domain-containing 1; C-terminus, carboxy-terminus; X- $\alpha$-gal, 5-bromo-4-chloro-3-indolyl- $\alpha$-D-galactopyranoside.

Thermo Fisher Scientific, Inc.), according to the manufacturer's protocol. At $48 \mathrm{~h}$ after transfection, co-localization of COMMD1 and lamin A in transfected cells was observed using a Leica TCS SP5 confocal microscope (Leica Microsystems, Inc.; magnification, x63) operated using Leica confocal software (LAS AF lite; version 2.0; Leica Microsystems, Inc.).

Co-immunoprecipitation. The pCMV-HA-COMMD1 vector was generated by inserting COMMD1 cDNA into the pCMV-HA vector (Clontech Laboratories, Inc.) with the EcoRI and $B g l \mathrm{II}$ restriction sites. Lamin A cDNA was inserted into the pCMV-Flag vector (Clontech Laboratories, Inc.) with the HindIII and NaeI restriction sites. The pCMV-Flag-lamin A, and pCMV-HA-COMMD1 or pCMV-HA plasmids were co-transfected into 293 cells using Lipofectamine ${ }^{\circledR} 2000$ (Invitrogen; Thermo Fisher Scientific, Inc.) according to the manufacturer's protocol. For immunoprecipitation, whole cell extracts of non-transfected or co-transfected cells were lysed with RIPA buffer (Thermo Fisher Scientific, Inc.). Equal amounts $(500 \mu \mathrm{g})$ of protein samples were incubated with $2 \mu \mathrm{g}$ antibody. The antibodies used in the co-immunoprecipitation experiment were as follows: Anti-HA and anti-Flag antibody for the overexpression plasmid and anti-COMMD1 or anti-lamin $\mathrm{A} / \mathrm{C}$ for endogenous co-immunoprecipitation. Normalmouse IgG (cat.no.sc-2025; Santa CruzBiotechnology, Inc.) and normal rabbit IgG (cat. no. sc-2027; Santa Cruz Biotechnology, Inc.) were used as control. Antibodies were incubated for $2 \mathrm{~h}$ at $4^{\circ} \mathrm{C}$. The antibody-protein complex was mixed with $100 \mu \mathrm{l}$ of a protein A/G-agarose suspension (cat. nos. 1134515 and 1243233; Roche Diagnostics, Inc.) for $24 \mathrm{~h}$ at $4^{\circ} \mathrm{C}$. After centrifugation at $12,000 \mathrm{x} \mathrm{g}$ for $20 \mathrm{sec}$ at $4^{\circ} \mathrm{C}$, the pellet was washed twice with wash buffer $1[50 \mathrm{mM}$ Tris- $\mathrm{HCl}$ ( $\mathrm{pH} 7.5), 150 \mathrm{mM}$ sodium chloride, $1 \%$ Nonidet $\mathrm{P}-40$, $0.5 \%$ sodium deoxycholate, and 1 tablet complete protein inhibitor cocktail/50 ml] and once each with wash buffer 2 (50 mM Tris- $\mathrm{HCl}, \mathrm{pH} 7.5,500 \mathrm{mM}$ sodium chloride, $0.1 \%$ Nonidet P-40, and $0.05 \%$ sodium deoxycholate) and wash buffer 3 (50 mM Tris-HCl, pH 7.5, 0.1\% Nonidet P-40 and $0.05 \%$ sodium deoxycholate) prior to resuspension in loading buffer. The suspension was denatured by heating for $5 \mathrm{~min}$ at $95-100^{\circ} \mathrm{C}$ and centrifuged at $12,000 \mathrm{x} \mathrm{g}$ for $10 \mathrm{~min}$ at $4^{\circ} \mathrm{C}$. The supernatant fraction was used for the identification of the co-precipitated proteins via western blotting, using the aforementioned procedure.

\section{Results}

Identification of COMMD1 as a novel lamin A-binding protein using yeast two-hybrid screening. To identify lamin A-binding proteins, a human skeletal muscle cDNA library was screened using the yeast two-hybrid system. Yeast cells expressing the C-terminus of lamin A (mRNA sequence 1,413-2,241) as bait were mated with yeast cells expressing the appropriate prey to make diploid yeast cells that were then streaked onto low-stringency TDO medium and high-stringency QDO medium to test for an interaction. At lower stringency, the colonies displayed $\mathrm{X}-\alpha-\mathrm{Gal}$ activity similar to that of the positive control (Fig. 1A). Following three rounds of selection, 9 colonies survived and were subjected to Sanger sequencing (Table SI). A fragment (Data S1) of the full sequence of COMMD1 (Data S2) gene was identified as a potential binding partner of lamin A via sequencing and a BLAST search (Fig. 1B). To validate this interaction, the COMMD1 prey was reintroduced into yeast cells and streaked onto QDO medium (Fig. 1C). Colonies growing on the QDO medium displayed X- $\alpha$-Gal activity similar to that of the positive control (Fig. 1D). These results suggested that COMMD1 may interact with lamin A. 

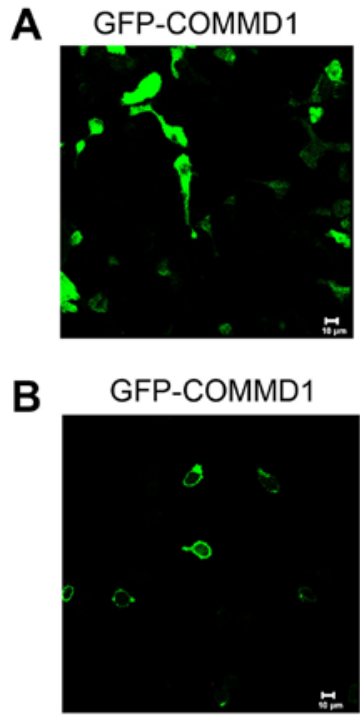

C

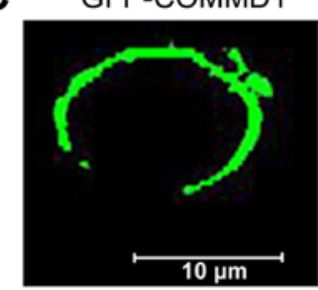

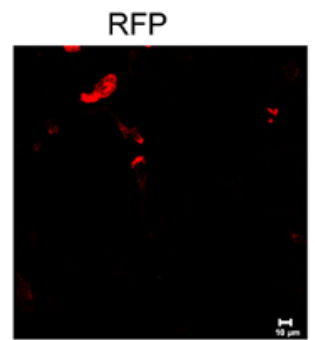

RFP-Lamin A

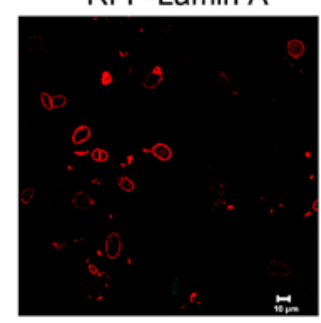

RFP-Lamin A

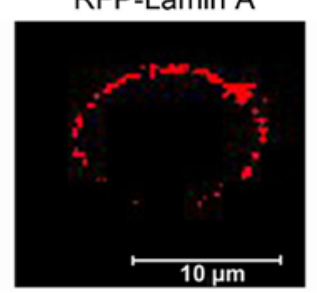

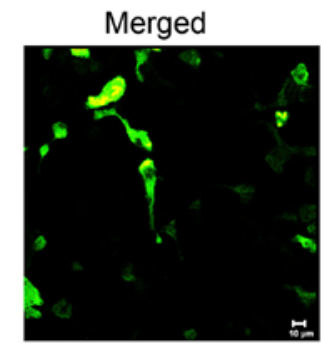

Merged

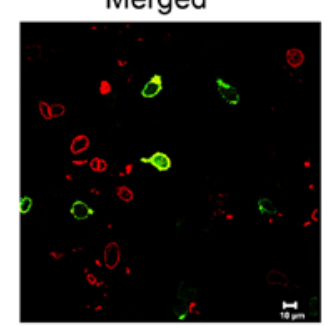

Merged

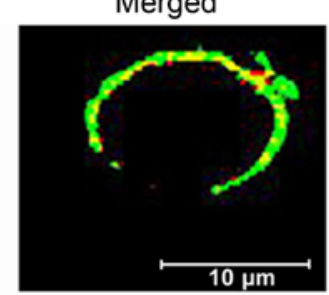

Figure 2. Co-localization of COMMD1 and lamin A in 293 cells. Co-transfection of 293 cells with (A) GFP-COMMD1 and pDsRed1-N1 (RFP) or (B) GFP-COMMD1 and RFP-lamin A. (C) Higher magnification view of cells co-transfected with GFP-COMMD1 and pDsRed1-N1-lamin A (RFP-lamin A). Cells were examined $24 \mathrm{~h}$ following transfection using confocal scanning laser microscopy. COMMD1, copper metabolism MURR1 domain-containing 1; GFP, green fluorescent protein; RFP, red fluorescent protein; RFP-lamin A, pDsRed1-N1-lamin A.

COMMD1 colocalizes with lamin A. To further verify the interaction between COMMD1 and lamin A, the subcellular localization of COMMD1 and lamin A was analyzed using fluorescence confocal microscopy. As shown in Fig. 2A, COMMD1 was predominantly cytoplasmic. The results of the co-transfection of 293 cells with GFP-tagged COMMD1 and RFP-tagged lamin A plasmids demonstrated that COMMD1 co-localized with lamin A in these cells (Fig. 2B and C).

COMMD1 physically interacts with lamin A. A co-immunoprecipitation assay was conducted to further test the interaction between COMMD1 and lamin A. Fig. 3A shows that COMMD1 interacted with lamin A. The interaction between endogenous COMMD1 and lamin A in 293 cells was evaluated by immunoprecipitation using anti-COMMD1 and anti-lamin $\mathrm{A} / \mathrm{C}$ antibodies followed by western blotting with an anti-lamin $\mathrm{A} / \mathrm{C}$ or anti-COMMD1 antibody. Following the co-transfection of 293 cells with the pCMV-Flag-lamin A plasmid, and the pCMV-HA-COMMD1 or pCMV-HA plasmid, immunoprecipitation was performed using an anti-HA antibody, followed by western blotting using an anti-Flag and anti-HA antibody. As shown in Fig. 3B, HA-COMMD1 was present in cell lysates from 293 cells transfected with both the pCMV-Flag-lamin A plasmid and the pCMV-HA-COMMD1 plasmid but not in cell lysates from 293 cells cotransfected with the pCMV-Flag-lamin A and pCMV-HA plasmids. Collectively, these results suggested that COMMD1 interacts with lamin A in vivo.

\section{Discussion}

In the present study, lamin A binding proteins were screened using the yeast two-hybrid system, and a novel interaction was identified between COMMD1 and lamin A. In addition to interacting with lamin A, COMMD1 was also observed to interact with prelamin A in other yeast two-hybrid screens (data not shown). The yeast two-hybrid system is a powerful method for the identification of proteins that interact with one another; however, it exhibits high false positive rates and involves non-physiological conditions (20). To overcome these drawbacks, the interaction between COMMD1 and lamin A was further validated using co-localization and co-immunoprecipitation experiments. Co-localization experiments using fluorescent confocal microscopy revealed that COMMD1 and lamin A co-localized to the nuclear envelope, which indicated the potential functional importance of their interaction. The interaction between COMMD1 and lamin A was also tested in endogenous and exogenous co-immunoprecipitation experiments. Collectively, the results of the present study revealed that COMMD1 physically interacts with lamin A.

COMMD1 is the best characterized member of the COMMD protein family, which consists of 10 subgroups (COMMD1-COMMD10) (21). A mutation in COMMD1 was originally identified as the genetic cause of canine copper toxicosis (22). Following that report, COMMD1 was found to be a pleiotropic protein that participates in several cellular processes, ranging from copper homoeostasis and sodium 
A
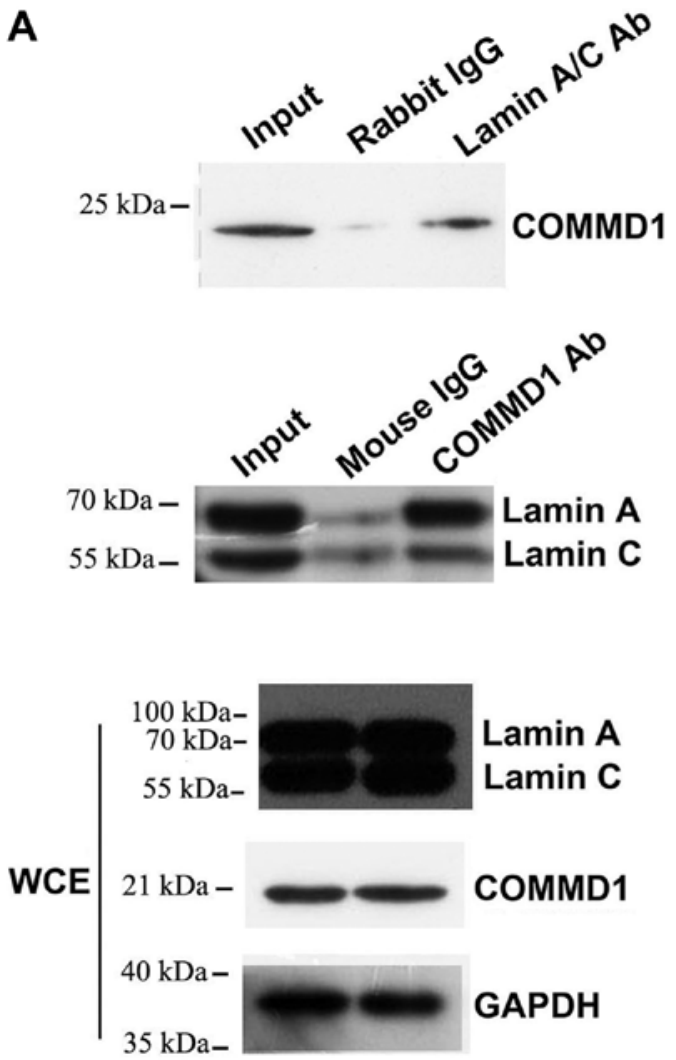

B
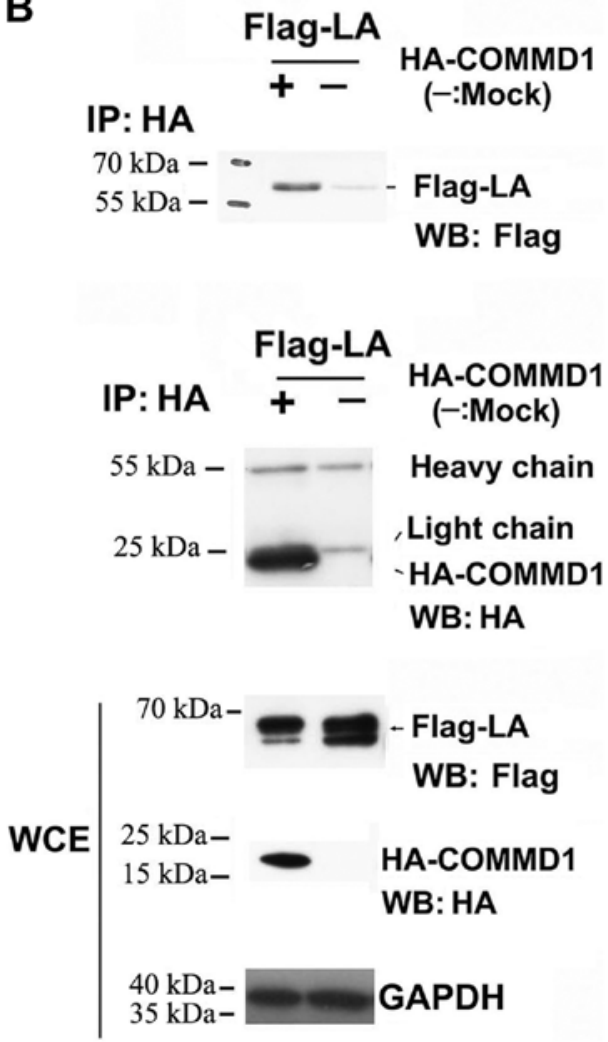

Figure 3. COMMD1 interacts with lamin A. (A) IP of 293 cell extracts with anti-lamin A/C or anti-COMMD1 antibodies, followed by western blotting with anti-COMMD1 and anti-lamin A/C antibodies. (B) Following co-transfection of 293 cells with pCMV-HA or HA-COMMD1 and Flag-lamin A, IP was conducted with an anti-HA antibody, followed by western blotting using an anti-Flag antibody. COMMD1, copper metabolism MURR1 domain-containing 1; HA, hemagglutinin; Ab, antibody; IgG, immunoglobulin G; WCE, whole cell extract; IP, immunoprecipitation; WB, western blotting.

transport, to the regulation of the $N F-\kappa B$ pathway and the expression of hypoxia-inducible factor- $1 \alpha$ (HIF-1 $\alpha)$-mediated genes (23). COMMD1 suppresses NF- $\kappa \mathrm{B}$ activity; however, the activation of $\mathrm{NF}-\kappa \mathrm{B}$ in Zmpste24-deficient mice resulted in premature aging (24). COMMD1 was found to regulate HIF-1 $\alpha$ activity during the adaptation to hypoxia by controlling HIF-1 $\alpha$ protein stability (25). Furthermore, the stabilization of HIF-1 $\alpha$ increased the lifespan of Caenorhabditis elegans $(26,27)$. Another COMMD1 binding partner, superoxide dismutase 1 , which is regulated by COMMD1 $(28,29)$, has also been shown to regulate normal cellular lifespan $(30,31)$. A G608G mutation in the LMNA gene or the loss of ZMPSTE24 activity resulted in the accumulation of unprocessed prelamin A in cells, which induced DNA damage and genomic instability, and led to premature aging $(8,9)$. DNA damage stimulated the relocalization of COMMD1 into the nucleoplasm and its interaction with alternate reading frame p14ARF (ARF) in humans and p19ARF in mice (32). ARF stabilizes the basal level of COMMD1 through K63-dependent polyubiquitination (32), which also promotes cellular senescence in mouse and human cells by regulating the $\mathrm{p} 53$ pathway $(33,34)$, and mediates resistance to cell cycle arrest in $L M N A^{-/-}$cells (35).

Lamin A is first synthesized as a prelamin A precursor with a conserved CAAX domain that is proteolytically processed by ZMPSTE24, removing the final 15 amino acids (36). The mature lamin A protein comprises a 28-residue positively charged globular head domain, a central $\alpha$-helical rod-shaped domain, a conserved nuclear localization signal and one immunoglobulin (Ig)-like structure domain formed of 116 residues at the C-terminus (37). The Ig-like domain was identified as an interaction hotspot that regulates interactions between lamin $\mathrm{A}$ and its binding partners $(37,38)$, its destabilization was found to affect most lamin A protein-protein interactions (3). The yeast two-hybrid results from the present study suggested that COMMD1 may interact with the C-terminus of lamin A. The interaction between COMMD1 and lamin A was verified using co-localization and co-immunoprecipitation assays. COMMD1 is highly conserved in vertebrates (39) and ubiquitously expressed in diverse eukaryotic tissues (40). COMMD1 predominantly localizes in the cytoplasm surrounding the nucleus $(41,42)$; however, a small fraction of COMMD1 can be found in the nucleus $(43,44)$. COMMD1 contains a COMM domain near its $\mathrm{C}$-terminus, which serves as a scaffold for protein-protein interactions (21), and has two highly conserved nuclear export signals, which are necessary and sufficient to induce nuclear export (44). In addition, in nucleocytoplasmic shuttling, COMMD1 may interact with lamin A at the nuclear envelope (44). The nuclear export of COMMD1 is important in the regulation of NF- $\kappa \mathrm{B}$ and HIF-1 activity in response to cellular stress (44). The interaction between COMMD1 and lamin A may also be involved in the regulation of the translocation of COMMD1 to the nucleoplasm from the perinuclear regions following DNA damage (32). In the present study, it was found that COMMD1 interacts with the C-terminus of 
lamin A; however, these results do not indicate if the interaction between COMMD1 and lamin $\mathrm{A}$ is direct or indirect. Co-immunoprecipitation and confocal fluorescence microscopy assays could not exclude the fact that other mediators are involved in this protein-protein interaction complex. As such, how and where COMMD1 interacts with lamin A requires further study.

In conclusion, to the best of our knowledge, the present study provided the first evidence that COMMD1 physically interacts with lamin A, suggesting possible roles for COMMD1 in cellular senescence and nucleocytoplasmic transport. Further studies are required to elucidate the precise mechanisms underlying the lamin A-COMMD1 interaction with regard to the aging process and laminopathies.

\section{Acknowledgements}

The authors would like to thank Professor Brian K. Kennedy (Buck Institute for Research on Aging), Professor Matt Kaeberlein (University of Washington), Professor Yousin Suh (Albert Einstein College of Medicine) and Professor Baohua Liu (Shenzheng University) for technical assistance and discussion.

\section{Funding}

The present study was supported by the National Natural Science Foundation of China (grant nos. 81671399 and 81170327), Ordinary University Innovation Team Construction Project of Guangdong Province (grant no. 2015KCXTD022), Unique Innovative Projects in Ordinary University of Guangdong Province (grant no. 2015KTSCX049), Dongguan International Science \& Technology Cooperation Project (grant no. 201650812001) and Scientific Research Fund of Guangdong Medical University (grant nos. 2XQ11009 and 2XK14030).

\section{Availability of data and materials}

The datasets used and/or analyzed during the current study are available from the corresponding author on reasonable request.

\section{Authors' contributions}

XL, WC and ZZ designed the study. ZJ drafted the manuscript. ZJ, JZ, QP, HZ and YY performed and analyzed the experiments. HC, WZ and XS analyzed the data. All authors read and approved the final manuscript.

\section{Ethics approval and consent to participate}

Not applicable.

\section{Patient consent for publication}

Not applicable.

\section{Competing interests}

The authors declare that they have no competing interests.

\section{References}

1. Dechat T, Pfleghaar K, Sengupta K, Shimi T, Shumaker DK, Solimando L and Goldman RD: Nuclear lamins: Major factors in the structural organization and function of the nucleus and chromatin. Genes Dev 22: 832-853, 2008.

2. Röber RA, Weber K and Osborn M: Differential timing of nuclear lamin $\mathrm{A} / \mathrm{C}$ expression in the various organs of the mouse embryo and the young animal: A developmental study. Development 105: 365-378, 1989.

3. Dittmer TA, Sahni N, Kubben N, Hill DE, Vidal M, Burgess RC, Roukos V and Misteli T: Systematic identification of pathological lamin A interactors. Mol Biol Cell 25: 1493-1510, 2014.

4. Rohani L, Johnson AA, Arnold A and Stolzing A: The aging signature: A hallmark of induced pluripotent stem cells? Aging Cell 13: 2-7, 2014.

5. Gonzalez JM, Pla D, Perez-Sala D and Andres V: A-type lamins and Hutchinson-Gilford progeria syndrome: Pathogenesis and therapy. Front Biosci (Schol Ed) 3: 1133-1146, 2011.

6. Andrés V and González JM: Role of A-type lamins in signaling, transcription, and chromatin organization. J Cell Biol 187: 945-957, 2009.

7. McCord RP, Nazario-Toole A, Zhang H, Chines PS, Zhan Y, Erdos MR, Collins FS, Dekker J and Cao K: Correlated alterations in genome organization, histone methylation, and DNA-lamin A/C interactions in Hutchinson-Gilford progeria syndrome. Genome Res 23: 260-269, 2013.

8. Musich PR and Zou Y: Genomic instability and DNA damage responses in progeria arising from defective maturation of prelamin A. Aging (Albany NY) 1: 28-37, 2009.

9. Singh S, Srivastava A, Srivastava P, Dhuriya YK, Pandey A, Kumar D and Rajpurohit CS: Advances in stem cell research-a ray of hope in better diagnosis and prognosis in neurodegenerative diseases. Front Mol Biosci 3: 72, 2016.

10. Meier I and Brkljacic J: The Arabidopsis nuclear pore and nuclear envelope. Arabidopsis Book 8: e0139, 2010.

11. Zuo B, Yang J, Wang F, Wang L, Yin Y, Dan J, Liu N and Liu L: Influences of lamin A levels on induction of pluripotent stem cells. Biol Open 1: 1118-1127, 2012.

12. Maggi L, Carboni N and Bernasconi P: Skeletal muscle laminopathies: A review of clinical and molecular features. Cells 5, 2016.

13. Korfali N, Wilkie GS, Swanson SK, Srsen V, de Las Heras J, Batrakou DG, Malik P, Zuleger N, Kerr AR, Florens L and Schirmer EC: The nuclear envelope proteome differs notably between tissues. Nucleus 3: 552-564, 2012.

14. Wong X, Luperchio TR and Reddy KL: NET gains and losses: The role of changing nuclear envelope proteomes in genome regulation. Curr Opin Cell Biol 28: 105-120, 2014.

15. Gonzalo S, Kreienkamp R and Askjaer P: Hutchinson-Gilford Progeria Syndrome: A premature aging disease caused by LMNA gene mutations. Ageing Res Rev 33: 18-29, 2017.

16. Navarro CL, De Sandre-Giovannoli A, Bernard R, Boccaccio I, Boyer A, Geneviève D, Hadj-Rabia S, Gaudy-Marqueste C, Smitt HS, Vabres P, et al: Lamin A and ZMPSTE24 (FACE-1) defects cause nuclear disorganization and identify restrictive dermopathy as a lethal neonatal laminopathy. Hum Mol Genet 13: 2493-2503, 2004.

17. Burtner CR and Kennedy BK: Progeria syndromes and ageing: What is the connection? Nat Rev Mol Cell Biol 11: 567-578, 2010.

18. Ghebre YT, Yakubov E, Wong WT, Krishnamurthy P, Sayed N, Sikora AG and Bonnen MD: Vascular aging: Implications for cardiovascular disease and therapy. Transl Med (Sunnyvale) 6, 2016.

19. Naito M, Omoteyama K, Mikami Y, Takagi M and Takahashi T: Suppression of lamin A/C by short hairpin RNAs promotes adipocyte lineage commitment in mesenchymal progenitor cell line, ROB-C26. Histochem Cell Biol 137: 235-247, 2012.

20. Kubben N, Voncken JW, Demmers J, Calis C, van Almen G, Pinto $\mathrm{Y}$ and Misteli T: Identification of differential protein interactors of lamin A and progerin. Nucleus 1: 513-525, 2010.

21. Burstein E, Hoberg JE, Wilkinson AS, Rumble JM, Csomos RA, Komarck CM, Maine GN, Wilkinson JC, Mayo MW and Duckett CS: COMMD proteins, a novel family of structural and functional homologs of MURR1. J Biol Chem 280: 22222-22232, 2005.

22. van De Sluis B, Rothuizen J, Pearson PL, van Oost BA and Wijmenga C: Identification of a new copper metabolism gene by positional cloning in a purebred dog population. Hum Mol Genet 11: 165-173, 2002. 
23. Bartuzi P, Hofker MH and van de Sluis B: Tuning NF- $\kappa B$ activity: A touch of COMMD proteins. Biochim Biophys Acta 1832: 2315-2321, 2013.

24. Osorio FG, Bárcena C, Soria-Valles C, Ramsay AJ, de Carlos F, Cobo J, Fueyo A, Freije JM and López-Otín C: Nuclear lamina defects cause ATM-dependent NF- $\mathrm{B}$ activation and link accelerated aging to a systemic inflammatory response. Genes Dev 26: 2311-2324, 2012

25. van de Sluis B, Muller P, Duran K, Chen A, Groot AJ, Klomp LW, Liu PP and Wijmenga C: Increased activity of hypoxia-inducible factor 1 is associated with early embryonic lethality in Commd1 null mice. Mol Cell Biol 27: 4142-4156, 2007.

26. Mehta R, Steinkraus KA, Sutphin GL, Ramos FJ, Shamieh LS, Huh A, Davis C, Chandler-Brown D and Kaeberlein M: Proteasomal regulation of the hypoxic response modulates aging in C. elegans. Science 324: 1196-1198, 2009.

27. Leiser SF, Begun A and Kaeberlein M: HIF-1 modulates longevity and healthspan in a temperature-dependent manner. Aging Cell 10: 318-326, 2011.

28. Vonk WI, Kakkar V, Bartuzi P, Jaarsma D, Berger R, Hofker MH, Klomp LW, Wijmenga C, Kampinga $\mathrm{HH}$ and van de Sluis B: The Copper metabolism MURR1 domain protein 1 (COMMD1) modulates the aggregation of misfolded protein species in a client-specific manner. PLoS One 9: e92408, 2014.

29. Vonk WI, Wijmenga C, Berger R, van de Sluis B and Klomp LW: $\mathrm{Cu}, \mathrm{Zn}$ superoxide dismutase maturation and activity are regulated by COMMD1. J Biol Chem 285: 28991-29000, 2010.

30. Blander G, de Oliveira RM, Conboy CM, Haigis $M$ and Guarente L: Superoxide dismutase 1 knock-down induces senescence in human fibroblasts. J Biol Chem 278: 38966-38969, 2003

31. Sun X, Komatsu T, Lim J, Laslo M, Yolitz J, Wang C, Poirier L Alberico T and Zou S: Nutrient-dependent requirement for SOD1 in lifespan extension by protein restriction in Drosophila melanogaster. Aging Cell 11: 783-793, 2012.

32. Huang Y, Wu M and Li HY: Tumor suppressor ARF promotes non-classic proteasome-independent polyubiquitination of COMMD1. J Biol Chem 283: 11453-11460, 2008.

33. Larsson LG: Oncogene- and tumor suppressor gene-mediated suppression of cellular senescence. Semin Cancer Biol 21: $367-376,2011$
34. Evan GI and d'Adda di Fagagna F: Cellular senescence: Hot or what? Curr Opin Genet Dev 19: 25-31, 2009.

35. Nitta RT, Smith CL and Kennedy BK: Evidence that proteasome-dependent degradation of the retinoblastoma protein in cells lacking A-type lamins occurs independently of gankyrin and MDM2. PLoS One 2: e963, 2007.

36. Barrowman J, Wiley PA, Hudon-Miller SE, Hrycyna CA and Michaelis S: Human ZMPSTE24 disease mutations: Residual proteolytic activity correlates with disease severity. Hum Mol Genet 21: 4084-4093, 2012.

37. Liu B and Zhou Z: Lamin A/C, laminopathies and premature ageing. Histol Histopathol 23: 747-763, 2008.

38. Goldman RD, Goldman AE and Shumaker DK: Nuclear lamins: Building blocks of nuclear structure and function. Novartis Found Symp 264: 3-16; discussion 16-21, 227-230, 2005.

39. Maine GN and Burstein E: COMMD proteins and the control of the NF kappa B pathway. Cell Cycle 6: 672-676, 2007.

40. Klomp AE, van de Sluis B, Klomp LW and Wijmenga C: The ubiquitously expressed MURR1 protein is absent in canine copper toxicosis. J Hepatol 39: 703-709, 2003.

41. Lian M and Zheng X: HSCARG regulates NF-kappaB activation by promoting the ubiquitination of RelA or COMMD1. J Biol Chem 284: 17998-18006, 2009.

42. Drevillon L, Tanguy G, Hinzpeter A, Arous N, de Becdelièvre A, Aissat A, Tarze A, Goossens M and Fanen P: COMMD1-mediated ubiquitination regulates CFTR trafficking. PLoS One 6: e18334, 2011.

43. de Becdelièvre A, Rocca J, Aissat A, Drévillon L, Moutereau S, Le Gouvello S, Hinzpeter A, Tarze A and Fanen P: COMMD1 modulates noxious inflammation in cystic fibrosis. Int J Biochem Cell Biol 45: 2402-2409, 2013.

44. Muller PA, van de Sluis B, Groot AJ, Verbeek D, Vonk WI, Maine GN, Burstein E, Wijmenga C, Vooijs M, Reits E and Klomp LW: Nuclear-cytosolic transport of COMMD1 regulates NF-kappaB and HIF-1 activity. Traffic 10: 514-527, 2009.

This work is licensed under a Creative Commons Attribution-NonCommercial-NoDerivatives 4.0 International (CC BY-NC-ND 4.0) License. 